Anschließend wird in Kapitel 3 gegenübergestellt, was anstelle von Anthraxfunden in den USA und ABC-Waffen im Irak hätte berichtet werden müssen: Das hätten nach Meinung der Autorin in prominenter Platzierung und Gewichtung die Haushaltsprobleme der USA sein sollen, der „Patriot Act“, die Umstände der Enron-Pleite, die Behandlung der Gefangenen in Guantanamo Bay („ausgeblendete Realität“) etc. Mit quasiagitatorischem Ton lässt sich die Autorin ein auf die Verwurzelung der aktuellen Bush-Politik in (neo-)konservativem Gedankengut. Sie präsentiert Strategiepapiere aus zahllosen Internetquellen. Die Darstellung ufert aus in polemischen Nachzeichnungen einzelner politischer, diplomatischer und militärischer Schritte. Illustriert wird dies mit einer unsystematischen Sammlung von Medienzitaten nach Reden von Präsident Bush und Außenminister Powell. Darin und in einer methodenfreien Internetrecherche in Archiven von ausgewählten US-, britischen und deutschen Printmedien erschöpfen sich die medienwissenschaftlich relevanten Aspekte des Hauptteils.

Als Conclusio in Kapitel 4 wird der Erfolg der politischen Planung, ihrer materiellen und kommunikativen Umsetzung und der Irreführung der Weltöffentlichkeit präsentiert. Die USA hätten zur „gewaltsamen Durchsetzung eines unilateralen Machtanspruches" auch die europäischen Länder und ihre Medien manipulativ für sich gewonnen. Die ausgeklügelten Bedrohungsszenarien, so das politische Plädoyer des Buches, dürften trotz realer Terrorgefahren die Bürgerrechte nicht im Kern beschneiden.

Was an der Quellenarbeit auffällt, ist der sträfliche Verzicht auf die Gegenprobe. Unverhältnismäßig wenige Bush-kritische Darstellungen in den amerikanischen Medien werden überhaupt erwähnt. Die Medienkritik in den US-Tageszeitungen und Magazinen sowie in den wissenschaftlichen Periodika und Publikationen wird kläglich vernachlässigt. Völlig ausgeblendet ist der Forschungsstand über Krisen und Kriege gleichsam als Ausnahme- und Routinesituation im Verhältnis von Politik, Medien und Politikadressaten in den USA. Das liegt aufgearbeitet vor. Selbst die offensichtliche Medienkritik innerhalb der Medien sowie die zu Bestsellern gelangten Bücher von investigativen US-Journalisten finden nicht statt. Sie hatten gerade in den USA einen reduplizierten Wirkungsgrad: bei den Millionen Lesern und bei den Medien, die sie breit thematisierten. Hier könnte man der Methodik des Buches folgend von einer ausgeblendeten Realität sprechen.

Was den Text so sperrig macht, ist eine befremdliche Metaphorik. Die Autorin bedient sich für Kapitel- und Zwischenüberschriften in alttestamentarischen Zitaten sowie Werbeslogans des deutschen Privatfernsehens und des Films. Einzelne Propaganda-Phasen der USRegierung z. B. werden „Schöpfungstage“ genannt. Weitere Leseprobe: „Powered by Emotion - erste Lageanalysen“, „A Fatal Attraction“, „Die üblichen Verdächtigen“ oder „Und er sah, dass es gut war". Wäre das Buch nur Monate später entstanden (fertig gestellt im Mai 2004), hätten unweigerlich die massiven Medienveröffentlichungen über die Manipulationen der Bush-Regierung und die Selbstreinigungsprozesse, in- und externe Revisionen der Medienberichterstattung einfließen müssen. Das lenkt Wasser auf die Mühlen der Kritiker, die eine synchrone wissenschaftliche Beschäftigung mit aktuellen politischen und Medienereignissen als konjunkturelle respektive modische Erscheinungen qualifizieren. Einen Beitrag zur Strukturdebatte um politische Kommunikation in Krisen- und Kriegszeiten hat dieses Buch nicht geleistet.

Christoph Rybarczyk

\section{Jörg Hagenah}

\section{Sportrezeption und Medienwirkung}

Eine dynamisch-transaktionale Analyse der Beziehungen zwischen Sportkommunikatoren und -rezipienten im Feld des Mediensports

München: Verlag Reinhard Fischer, 2004 $180 \mathrm{~S}$.

(Reihe Medien-Skripten; 41)

ISBN 3-88927-348-3

Die Zeiten akademischer Ignoranz und wissenschaftlicher Abstinenz beim Thema Mediensport sind längst vorbei. Die wachsende gesellschaftliche Akzeptanz des Genres Sports korrespondiert mit der steigenden publizistischen Relevanz des Sujets Sportberichterstattung. Diese Entwicklung hat auch $\mathrm{zu}$ vielfältigen Forschungsaktivitäten geführt, wie eine Reihe einschlägiger Monographien und Anthologien vor allem seit den 90er Jahren dokumentiert. Meistens geht es dabei um Grundlagenstudien 
für die Bereiche Kommunikator-, Medien- und Aussagenforschung. Ein absolutes Schattendasein der sportpublizistischen Analysepraxis stellt dagegen immer noch der Bereich der $\mathrm{Pu}$ blikums- und Wirkungsforschung dar, sieht man einmal davon $a b$, dass die quantitative Nutzungsforschung je nach Medium telemetrisch oder über Befragungen erhebt, wer, wann, wie häufig und wie lange welches Sportangebot rezipiert. Die qualitative Nutzenforschung sowie Fragen nach Motiven und Effekten sind bislang fast immer ignoriert worden. Und auch die Forderungen, die traditionellen und neueren Ansätze der Publikumsund Wirkungsforschung für die Sportberichterstattung zu überprüfen, blieben bislang weitestgehend ungehört. Die sportbezogene empirische Wirkungsforschung steht sowohl in der Kommunikations- als auch in der Sportwissenschaft klar im Abseits. Komplexität und Kompliziertheit des Gegenstandsbereichs sind vermutlich wichtige Gründe dafür, dass bislang national wie international nur wenige Fallstudien hierzu durchgeführt werden konnten.

Vor dem Hintergrund dieses quantitativ wie qualitativ äußerst übersichtlichen Forschungsstandes muss die Studie „Sportrezeption und Medienwirkung" von Jörg Hagenah, die als Dissertationsschrift an der Sportwissenschaftlichen Fakultät der Universität Leipzig angenommen wurde, als anerkennenswerter Beitrag gesehen werden, ein wenig Licht in diese sportpublizistische „Black Box“ zu bringen. Dass der Autor dabei seine Untersuchungen auf das dynamisch-transaktionale Modell von Klaus Schönbach und Werner Früh und damit auf einen der anspruchsvollsten Wirkungsansätze stützt, erscheint mutig und muss mit Blick auf die dabei eruierten Ergebnisse auch als ein wenig überambitioniert eingestuft werden. Bereits der Untertitel ist irreführend, denn die versprochene Analyse der Beziehungen zwischen Sportkommunikatoren und -rezipienten im Feld des Mediensports wird nur teilweise eingelöst: Sportjournalisten als die eine wichtige Seite dieses Modells werden im empirischen Teil überhaupt nicht untersucht und spielen bei der theoretischen Verortung ebenfalls nur eine rudimentäre Rolle. Es wäre sicher angemessener gewesen, im Untertitel den Begriff der Sportkommunikatoren durch den Terminus Sportkommunikate zu ersetzen. Der Autor selbst konzediert zurecht einige Male die „Rezeptionslastigkeit“ seiner Studie (S. 70, S. 170).
Im einleitenden Kapitel „Sportrezeption und Medienwirkungen" wird die Bedeutung des Problemfelds „Mediensport“ auf der Basis ausgewählter Beispiele angerissen. Die angeführten Belege sind aber teilweise - wie etwa „Sportstunden pro Jahr bis 1996“ oder „Zuschauerzahlen beim Tennis von 1986“ - genauso überholt wie die daraus abgeleiteten Interpretationen. Unter der Überschrift „Das mediale Sportsystem“ werden auf der Makro-, Meso- und Mikroebene die vier gesellschaftlichen Teilbereiche Sport, Wirtschaft, Medien und Publikum systematisch durchkonjugiert. Dies jedoch als eine „systemtheoretische Herangehensweise" (S. 15) zu etikettieren, ohne die entsprechenden Codes, Programme oder Kommunikationsmedien der identifizierten „Subsysteme" auch nur zu erwähnen, erscheint fragwürdig. Dagegen bieten die nachfolgend beschriebenen Kopplungen zwischen den genannten vier Teilbereichen einen pointierten Überblick über spezifische Abhängigkeitskonstellationen (S. 22-24). Dies gilt ebenso für die ergänzende Synopse zu Zielen und möglichen Transaktionen der Akteure im Mediensport (S. 30). Kleinere Rechercheschwächen weist die Aufarbeitung des Forschungsstands zum Thema Mediensport auf, weil vor allem beim Unterkapitel „Inhaltsanalysen“ und partiell auch beim Unterkapitel „Publikum“ einige Titel und Studien nicht zur Kenntnis genommen worden sind. Die Darstellung verschiedener theoretischer Ansätze der allgemeinen und sportbezogenen Publikums- und Medienwirkungsforschung ist recht komprimiert, beinhaltet aber die wesentlichen Aspekte und ordnet die wenigen empirischen Sport-Wirkungs-Untersuchungen und ihre Ergebnisse sinnvoll zu. Da aber bislang nur wenige empirische Studien vorliegen, wäre eine ausführlichere Besprechung und vor allem intensivere Bewertung durch den Verfasser von Interesse gewesen, vor allem für den Leser, der die genannten Arbeiten nicht bzw. nur ausschnitthaft kennt. Die Diskussion des für die vorgelegte Publikation forschungsleitenden dynamisch-transaktionalen Modells und seine sportspezifische Einordnung ist für die Rezeptions-Intra-Transaktionen ansprechend gelungen, weil hier vier relevante Aspekte („Sport-TV-Motive“, „Sensation Seeking “, „Involvement" und „soziodemographische Variablen“) nachvollziehbar herausgearbeitet wurden. Dieser Grad an Differenziertheit nimmt dann aber bei den präsen- 
tierten sportmedialen Inter-Transaktionen deutlich $\mathrm{ab}$ und ist für die nur noch andiskutierten Kommunikator-Transaktionen kaum noch zu erkennen.

Die zentrale Frage der Studie, ob mediale Botschaften eher medial gesteuert rezipiert oder rezeptionsmotivational abhängig bewertet werden, geht der Autor in einem recht aufwändigen Untersuchungsdesign nach. Drei Teiluntersuchung mit insgesamt 979 Probanden wurden durchgeführt. Rätselhaft erscheinen die vielen zahlenmäßigen Widersprüche in der Tabelle, in der die Zusammensetzungen der drei Stichproben aufgelistet ist (S. 78). Schon die einfache Addition innerhalb verschiedener Merkmale führt häufig zu unterschiedlichen Probandenzahlen einer Stichprobe. Zudem wäre bei der gesamten Beschreibung des Untersuchungsdesigns mehr sprachliche Präzision nützlich gewesen. Dass die Stichprobengrößen zahlenmäßig sowie vom Alter und der Tätigkeit der Testteilnehmer stark variieren, ist aus organisatorischen Gründen nachvollziehbar, hätte spätestens aber bei der Ergebnisinterpretation zu deutlich relativierteren Einschätzungen bezüglich der Datenlagen und ihrer Aussagekraft führen können.

Die Probanden der ersten beiden Teiluntersuchungen wurden mit gleich layouteten, aber unterschiedlich kommentierten Artikeln zu zwei Stabhochsprung-Wettkämpfen anlässlich der Leichtathletik-WM in Sevilla konfrontiert und befragt. Bei den manipulierten Fassungen gab es zwei Versionen, die die Leistungen der beiden deutschen Springer als großen Erfolg darstellten, zwei weitere, die die Leistung als sportliche Niederlage bewerteten und eine Version, die als neutrale Kontrollnachricht dienen sollte. Die Probanden der dritten Teiluntersuchung schauten sich vier Versionen eines Skispringens in Bischofshofen auf RTL und Eurosport an, die hinsichtlich des Kommentars (mit bzw. ohne Kommentar) und bezüglich des Senderlogos variiert wurden. Wozu der Logowechsel dienen soll, bleibt offen. Und die Prüfung eines Sportevents ganz ohne Ton, sozusagen in einer „sterilen“ Bildfassung, ist derart atypisch, dass die hierdurch gewonnenen Ergebnisse sicher hinter den selbst gesteckten Ansprüchen zurückbleiben müssen. Verschiedene Variationen des Kommentartextes hätten zweifellos zu aussagekräftigeren Befunden geführt.

Insgesamt wurden 13 verschiedene Messin- strumente eingesetzt, um mögliche Einflussvariablen für die Rezeption von Mediensport zu eruieren. Dabei greift der Autor zum Teil auf bereits vorliegende Skalen und Items zurück, überprüft deren Nützlichkeit für seine Forschungsfrage und entwickelt gegebenenfalls sinnvollere Kriterien. Die Ergebnisse verschiedener Faktoren-, Varianz- und linearer Regressionsanalysen zur Identifizierung maßgeblicher Einflussfaktoren und ihrer jeweiligen Beziehungen untereinander werden in mehr als 50 Tabellen präsentiert. Interessante Hinweise finden sich in dem entwickelten intratransaktionalen Modell der Sportrezeption (S. 123) sowie in den inter-transaktionalen Modellen der Sportrezeption „Print“ und „TV“.

Der Autor ermittelt für den Konsum von Sport-Printmedien das Motiv „Lernen/Informationssuche" und für die Nutzung von Sport im Fernsehen die „parainteraktive Emotionssuche" als besonders relevante Einflussgrößen. Der wahrgenommene Unterhaltungswert einer Medienbotschaft ist für das Weiterverfolgen einer Sendung bedeutsam, aber scheinbar irrelevant für die zukünftige Rezeption ähnlicher Angebote. Die Bewertungen der geprüften Beiträge durch die Befragten werden sowohl von der Rezeptionsmotivation als auch von der Kommentierung beeinflusst. Schließlich ließen sich sowohl Beitrags- als auch Aktivationseffekte nachweisen. Die Studie liefert Hinweise für einen Zusammenhang zwischen persönlichen Dispositionen, daraus resultierenden Rezeptionsmotiven und sich ergebenden Rezeptionsmodalitäten beim Verfolgen und Einschätzen von Sportmedienangeboten. In der Ergebniszusammenfassung formuliert der Autor selbst Einschränkungen zur Aussagekraft der gewonnenen Befunde (S. 127), hinterfragt die Kurz- bzw. Langfristigkeit der ermittelten Effekte (S. 129) und warnt zu Recht vor einer Überinterpretation der Ergebnisse (S. 169). Sein Plädoyer, die hypothesegenerierenden Resultate in künftigen Studien zu überprüfen und die entwickelten Modelle empirisch weiter abzusichern, ist folgerichtig, sollte aber um die Variante der Modifikation zumindest optional erweitert werden. Der Autor stellt in seinen Schlussbetrachtungen eine Reihe sinnvoller Fragen, die zu Impulsgebern für künftige Forschungsprojekte werden könnten. Welchen Einfluss Geschlecht, Alter, Bildung, spezielles sportliches Fachwissen oder eigenes Sporttreiben auf die Rezeption von Sportmedienange- 
boten haben, konnten hier nicht geklärt werden. Und die ebenfalls vernachlässigte Berücksichtigung der Kommunikatorseite könnte ebenso einen weiteren interessanten Ansatzpunkt bieten.

Die Studie von Jörg Hagenah stellt mit allen beschriebenen Defiziten und Stärken einen mutigen Schritt in die richtige Richtung zu einem theoretisch und vor allem empirisch bislang kaum bearbeiteten Forschungsfeld dar. Nicht nur, aber auch in Ermangelung vergleichbarer Analysen wird man die Untersuchung aufmerksam zur Kenntnis nehmen müssen, wenn man sich auf das komplizierte Gebiet der sportmedialen Publikums- und Wirkungsforschung wagt.

Michael Schaffrath

\section{Martin R. Schütz}

\section{Journalistische Tugenden}

Leitplanken einer Standesethik

Wiesbaden: Westdeutscher Verlag, 2003. $250 \mathrm{~S}$.

\section{ISBN 3-531-14088-4}

Dies ist ein notwendiges, interessantes, hilfreiches Buch über journalistische Ethik. Solch ein Buch kann- jedenfalls zurzeit - wohl nur ein Schweizer schreiben; solch ein Buch kann wohl nur ein Schweizer Journalist schreiben. Das sind Stereotype, aber sie stimmen.

In der Schweiz macht man sich immer noch Gedanken darüber, was wir verlieren, wenn der Journalismus seine Maßstäbe preisgibt und vollends zu einer Branche von Unterhaltungskünstlern wird, in der nur der (vermeintliche) Publikumserfolg zählt. Das ist in Deutschland anders - auch wenn der Nachbar allmählich dieselben strukturellen Probleme bekommt, die sich hier wie dort (Fall Borer) als affärenanfällig erweisen. Doch bei uns regt sich kaum noch jemand darüber auf, dass die Nachrichtenmedien zunehmend infotainisiert werden zumal (immer mehr) Medien- und Kommunikationswissenschaftler eilfertig dabei mitwirken, die Grenzen zur Unterhaltung zur Disposition zu stellen, weil sie verzopft, frauenfeindlich und generell publikumsfremd seien. Aus demselben Fach - freilich mit dezidiert „philosophischem " Hintergrund - stammen andererseits auch jene Ethikhelfer, die auf Letztbegründungen (auch) beim einschlägigen Diskurs im Journalismus pochen und vor praktischen Problemen in eine verquaste „Metaethik“ emigrieren.

Der Journalist Martin Schütz, Redakteur bei der Schweizerischen Depeschenagentur, tut in seiner Dissertation, mit der er an der Universität Basel promoviert wurde, genau das $\mathrm{Ge}$ genteil von all dem. Er stellt sich den Herausforderungen und den Fallen, die auf denjenigen lauern, der hier konkret wird. Allen Ernstes, und das gleich zu Anfang, verspricht er, nicht für moralische Schwierigkeiten im Journalismus sensibilisieren, sondern Lösungsansätze aufzeigen und Orientierungshilfen für den Alltag geben zu wollen. Das soll nicht auf eine geschlossene Systematik für eine Standesethik hinauslaufen; der Autor bekennt sich offen zum Applikationscharakter seines Unternehmens. Und das immerhin in einer Doktorarbeit, also einer Textsorte sui generis.

Martin Schütz arbeitet sehr bewusst mit der Metapher der Brücke - zwischen der journalistischen Praxis, die er erfahren, und der praktischen Philosophie, die er offenbar gründlich studiert hat. In der Mitte der Brücke bezieht er seinen persönlichen Standpunkt, den er geradezu bekennerhaft immer wieder deutlich macht. Von hier aus hat er den Überblick, um sein ehrgeiziges Projekt zu realisieren, zweieinhalb tausend Jahre Philosophiegeschichte für eine moderne Tugendlehre des Journalismus zu nutzen.

Dabei hat Schütz vor allem drei wichtige Helfer: den römischen Philosophen Seneca, den US-amerikanischen Sozialphilosophen Michael Walzer und den tschechisch-deutschen Journalisten Egon Erwin Kisch. Ein Seneca-Zitat bildet die Grundmelodie der Arbeit - und die finde ich ausgesprochen sympathisch und hilfreich für die Ethik-Lehre: Dass die Philosophie zugleich eine theoretische und praktische Wissenschaft ist (was nicht allen „Medienethikern“" klar zu sein scheint), und dass man nur dann für den Einzelfall gewappnet ist, wenn einem vernünftige Möglichkeiten sozusagen für alle Fälle vermittelt worden sind.

Der Rekurs auf Walzer nutzt die Einsicht, dass sich Gesellschaftskritik - und dazu gehören Medienkritik und Medienethik - auf Werte beziehen muss, die in einer Gesellschaft anerkannt sind und von den Menschen geteilt werden. Kisch schließlich liefert mit seinem professionellen und ethischen Versagen beim „Debüt am Mühlenfeuer“ die konkrete Folie, 